\title{
Tolérancement fonctionnel optimisé par la méthode des dispersions
}

\author{
Nassima Medjadi-Cheikh ${ }^{1, a}$, SaÏd Hamou $^{1}$, Djezouli Moulai-Khatir ${ }^{1}$, \\ Abdelmadjid Cheikh ${ }^{1}$ et Jean-Marc Linares ${ }^{2}$ \\ 1 LAT, Département de Mécanique, Faculté de Technologie, Université Abou Bekr Belkaid, BP 230, Tlemcen 13000, Algérie \\ 2 Institut des Sciences du Mouvement/GIBO CNRS UMR 6233, Département de Génie mécanique et Productique, \\ IUT d'Aix-en-Provence, Avenue Gaston Berger, 13625 Aix-en-Provence Cedex 1, France
}

Reçu le 30 décembre 2009, accepté le 5 novembre 2010

Résumé - Cet article présente l'optimisation du tolérancement fonctionnel par la méthode des dispersions, celle-ci a fait ces preuves dans l'optimisation des cotes de fabrication. Dans ce travail la méthode des dispersions inconnues est combinée avec la méthode du transfert minimum pour la modélisation de l'automatisation de la simulation des exigences fonctionnelles d'un assemblage mécanique. La simulation consiste en l'extraction automatique des chaînes fonctionnelles d'une part, et en l'optimisation des dispersions d'autre part. Enfin, les cotes fonctionnelles sont synthétisées à partir des dispersions optimisées en terme de cotes moyennes et intervalles de tolérances centrés. Elles sont en adéquation avec l'écriture au sens de la norme ISO des cotes fonctionnelles.

Mots clés : Méthode des dispersions / optimisation / chaîne de cotes / tolérances fonctionnelles / synthèse / ISO

\begin{abstract}
Functional tolerancing optimised by dispersions method. This paper presents the optimization of functional tolerancing by dispersions method, this later has made this evidence in manufacturing dimensions optimization. In this work the dispersions method on the basis of unknown variables is combined with the minimal transfer method for automatically the modelling of the functional requirements of a mechanical assembly. The simulation is constructed around two procedures. The first procedure executes the extraction of the functional requirements, the second procedure results in the optimization of the dispersions. The functional dimensions are synthesized by uses of the optimised values of the dispersions to compute the mean values and tolerances of the functional dimensions in the chains. They are in adequacy with the writing within the meaning of the ISO standard of the functional dimensions.
\end{abstract}

Key words: Dispersions method / optimization / dimension chains / functional tolerances / synthesis / ISO

\section{Introduction}

L'expérience a montré que la détermination des tolérances des pièces en conception mécanique nécessite des choix importants qui influent sur le mode de fabrication et le prix de revient des pièces. Si l'on ne définit pas un tolérancement optimal, on risque soit d'augmenter les coûts de fabrication par des tolérances trop sévères ou mal placées, soit de produire des pièces qui ne conviendront pas dans l'assemblage ou encore des assemblages peu fiables en fonctionnement. En effet, les décisions prises

\footnotetext{
a Auteur pour correspondance :

nm_cheikh@yahoo.fr, acheikh@mail.univ.tlemcen.dz
}

durant la phase de conception induisent près de $70 \%$ du coût total de réalisation du produit [1]. Pour cela il faut optimiser les moyens de fabrication pour produire des pièces de bonne qualité et à moindre coût. L'objectif de l'optimisation des tolérances de conception est de minimiser le coût total de fabrication de toutes les tolérances tout en respectant les exigences fonctionnelles. Actuellement les outils, les méthodes et les théories associées au tolérancement restent encore très pauvres [2]. Le concepteur trouve quelques systèmes dédiés [3] mais peu de solutions intégrées. Dans cette offre, les outils proposés traitent essentiellement de l'analyse des tolérances [4]. L'optimisation des tolérances fonctionnelles a longuement 


\section{Nomenclature}

\begin{tabular}{|lc|}
\hline Symbole & Désignation \\
\hline$\Delta l$ & Dispersion totale \\
$C f$ & Cote fonctionnelle \\
$C C$ & Cote condition \\
$l$ & Surface de départ \\
$m$ & Surface d'arrivée \\
$I T$ & Intervalle de tolérance \\
$C S$ & Cote standard \\
\hline
\end{tabular}

été traitée par la méthode vectorielle non rationnelle [5], qui a pour inconvénient de donner des tolérances serrées, donc un coût de fabrication élevé. La méthode des dispersions appelée méthode $\Delta l$ introduite par Bourdet [6] représente une méthode efficace pour la simulation d'usinage $[7,8]$. Dans le cas de chaînes de cotes unidirectionnelles, les jeux sont tous modélisés par une seule composante de translation dont les intervalles de variation sont notées $\Delta l_{j}^{i}$ dans la méthode $\Delta l[9]$. Dans ce contexte, ce travail présente une modélisation de la simulation des tolérances de conception d'un assemblage mécanique par la méthode des dispersions qui est basée sur le principe du pire des cas. La méthode $\Delta l$ est combinée avec la méthode du transfert minimum introduite par Duret [10] pour automatiser la procédure d'extraction des chaînes de cotes fonctionnelles par un calcul matriciel. L'optimisation des dispersions est effectuée à l'aide de la méthode des dispersions inconnues $[1,11]$. Les dispersions optimisées sont utilisées pour calculer les intervalles de tolérances et les longueurs de simulation sont utilisées pour synthétiser les valeurs moyennes pour les cotes fonctionnelles.

\section{Modélisation de la simulation des conditions fonctionnelles}

\subsection{Principe de la méthode des dispersions}

Lors de l'usinage en série de la longueur $L$ d'une pièce, dans les mêmes conditions, on remarque pour un réglage donné, une variation dimensionnelle des pièces successivement usinées. On appelle donc dispersion globale ou dispersion totale, la différence entre la plus grande et la plus petite dimension relevée sur une série de pièces réalisées à partir d'un même réglage d'outil.

$$
\Delta l=L_{\operatorname{maxi}}-L_{\operatorname{mini}}
$$

Le modèle des $\Delta l$ introduit la notion de cotes de simulation $L i$ qui permet de situer dans chaque repère de la production, la surface d'appui et les différentes surfaces fabriquées. $\Delta l_{i}$ est la dispersion permise d'une cote de simulation $L i$ représentant la variation de position de la surface $i$ dans le repère fixe lié à la machine de production.

$$
\left(C f_{i, j}\right)_{\mathrm{moy}}=L_{j}-L_{i}
$$

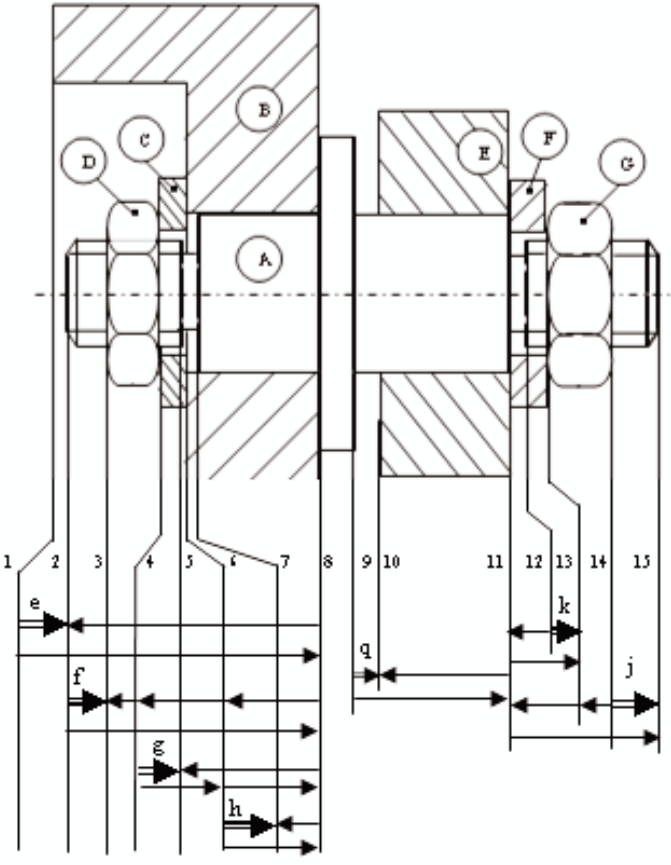

Fig. 1. Exemple d'assemblage.

\subsection{Matrice des dispersions}

L'exemple simplifié de la figure 1 [8], représente la modélisation de la simulation unidirectionnelle d'un assemblage de pièces. Toutes les surfaces sont modélisées par des points de substitutions [12] (situés à l'extérieur de la matière, ces derniers sont supposés représenter la géométrie réelle des pièces). Ils sont numérotés par ordre croissant de gauche à droite. Les pièces sont repérées par des lettres alphabétiques. L'assemblage est ensuite représenté sous forme d'une matrice de dispersions affectées aux surfaces des pièces [13]. Enfin, un calcul matriciel est effectué pour chaque condition fonctionnelle de l'assemblage par la méthode du transfert minimum.

$$
\begin{aligned}
e_{1,2} & =C f_{1,8}^{(\mathrm{B})}-C f_{2,8}^{(\mathrm{A})} \\
f_{2,3} & =C f_{2,8}^{(\mathrm{A})}-C f_{6,8}^{(\mathrm{B})}-C f_{4,6}^{(\mathrm{C})}-C f_{3,4}^{(\mathrm{D})} \\
g_{4,5} & =C f_{4,6}^{(\mathrm{C})}+C f_{6,8}^{(\mathrm{B})}-C f_{5,8}^{(\mathrm{A})} \\
h_{6,7} & =C f_{6,8}^{(\mathrm{B})}-C f_{7,8}^{(\mathrm{A})} \\
q_{9,10} & =C f_{9,11}^{(\mathrm{A})}-C f_{10,11}^{(\mathrm{E})} \\
k_{12,13} & =C f_{11,13}^{(\mathrm{F})}-C f_{11,12}^{(\mathrm{A})} \\
j_{14,15} & =C f_{11,15}^{(\mathrm{A})}-C f_{11,13}^{(\mathrm{F})}-C f_{13,14}^{(\mathrm{G})}
\end{aligned}
$$

Les conditions fonctionnelles sont les suivantes :

$e=2 \pm 0,25$ : Retrait de la tête de l'arbre A par rapport à la pièce $B$;

$f=4 \pm 0,75$ : Dépassement de la tête extérieure de filetage de l'arbre A par rapport à l'écrou D;

$g=k=2 \pm 0,5$ : Dépassements intérieurs suffisants des filetages pour la prise des écrous $\mathrm{D}$ et $\mathrm{G}$; 


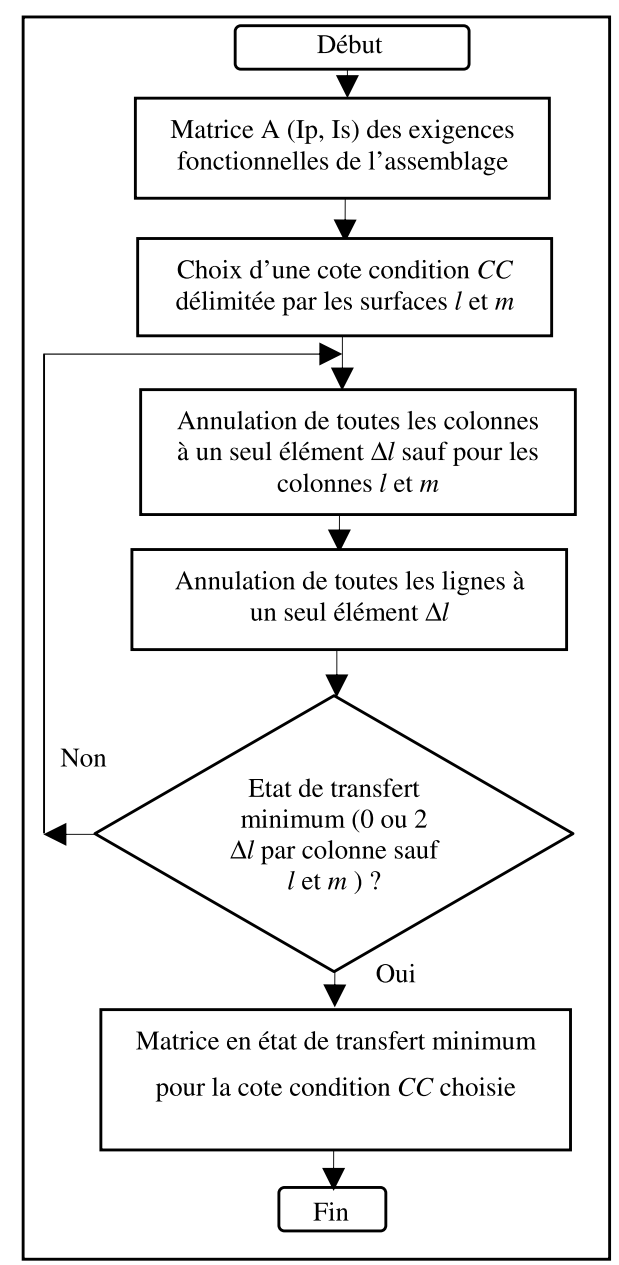

Fig. 2. Organigramme de la méthode du transfert minimum [14].

$h=2 \pm 0,5$ : Serrage entre les pièces A et B;

$j=4 \pm 1$ : Dépassement de la tête extérieure de filetage de l'arbre A par rapport à l'écrou $\mathrm{G}$;

$q=3 \pm 0,1$ : Jeux existants entre le galet $\mathrm{E}$ et ses pièces voisines $\mathrm{A}$ et $\mathrm{F}$.

L'assemblage est représenté sous forme d'une matrice de Is colonnes et de Ip lignes. Is représente l'indice de surface et Ip représente l'indice de pièces. Comme le montre le tableau 1, l'élément $A_{\mathrm{Is}, \mathrm{Ip}}$ de la matrice contient une valeur de dispersion uniquement lorsque la surface Is fait partie de la pièce Ip comme surface de contact ou surface terminale. Dans le cas contraire il est nul.

\section{Extraction des chaînes de cotes fonctionnelles par la méthode du transfert minimum}

Le principe de la méthode comme l'illustre la figure 2 est de reconnaître tout d'abord les surfaces qui délimitent une cote condition. Nous les notons $l$ et $m$. Nous procédons à l'annulation des dispersions uniques qui sont présentes dans toutes les colonnes, excepté celles qui se trouvent sur les colonnes $l$ et $m$. Nous procédons de la même manière pour les lignes, mais cette fois-ci sans exception bien sûr.

Nous répétons ces opérations jusqu'à l'aboutissement à un transfert minimum donné par 0 ou $2 \Delta l$ par colonne. Une fois qu'on est en transfert minimum, les cotes fonctionnelles qui participent à la réalisation des cotes conditions sont les cotes délimitées par les surfaces qui abritent les deux dispersions présentes sur la pièce (même ligne de la matrice).

\section{Optimisation par la méthode des dispersions inconnues}

L'application de la procédure d'extraction des chaînes de cotes fonctionnelles appliquée à l'assemblage a permis d'avoir les chaînes de tolérances associées suivantes :

$$
\begin{aligned}
\Delta e_{1,2}= & \sum \Delta l_{i}=\left(\Delta l_{2}^{(\mathrm{A})}+\Delta l_{8}^{(\mathrm{A})}\right)+\left(\Delta l_{1}^{(\mathrm{B})}+\Delta l_{8}^{(\mathrm{B})}\right) \\
\Delta f_{2,3}= & \sum \Delta l_{i}=\left(\Delta l_{2}^{(\mathrm{A})}+\Delta l_{8}^{(\mathrm{A})}\right)+\left(\Delta l_{6}^{(\mathrm{B})}+\Delta l_{8}^{(\mathrm{B})}\right) \\
& +\left(\Delta l_{4}^{(\mathrm{C})}+\Delta l_{6}^{(\mathrm{C})}\right)+\left(\Delta l_{3}^{(\mathrm{D})}+\Delta l_{4}^{(\mathrm{D})}\right) \\
\Delta g_{4,5}= & \sum \Delta l_{i}=\left(\Delta l_{5}^{(\mathrm{A})}+\Delta l_{8}^{(\mathrm{A})}\right)+\left(\Delta l_{6}^{(\mathrm{B})}+\Delta l_{8}^{(\mathrm{B})}\right) \\
& +\left(\Delta l_{4}^{(\mathrm{C})}+\Delta l_{6}^{(\mathrm{C})}\right) \\
\Delta h_{6,7}= & \sum \Delta l_{i}=\left(\Delta l_{7}^{(\mathrm{A})}+\Delta l_{8}^{(\mathrm{A})}\right)+\left(\Delta l_{6}^{(\mathrm{B})}+\Delta l_{8}^{(\mathrm{B})}\right) \\
\Delta q_{9,10}= & \sum \Delta l_{i}=\left(\Delta l_{9}^{(\mathrm{A})}+\Delta l_{11}^{(\mathrm{A})}\right)+\left(\Delta l_{10}^{(\mathrm{E})}+\Delta l_{11}^{(\mathrm{E})}\right) \\
\Delta k_{12,13}= & \sum \Delta l_{i}=\left(\Delta l_{11}^{(\mathrm{A})}+\Delta l_{12}^{(\mathrm{A})}\right)+\left(\Delta l_{11}^{(\mathrm{F})}+\Delta l_{13}^{(\mathrm{F})}\right) \\
\Delta j_{14,15}= & \sum \Delta l_{i}=\left(\Delta l_{11}^{(\mathrm{A})}+\Delta l_{15}^{(\mathrm{A})}\right)+\left(\Delta l_{11}^{(\mathrm{F})}\right. \\
& \left.+\Delta l_{13}^{(\mathrm{F})}\right)+\left(\Delta l_{13}^{(\mathrm{G})}+\Delta l_{14}^{(\mathrm{g})}\right)
\end{aligned}
$$

À partir des chaînes de tolérances exprimées en fonction des dispersions, on forme une matrice globale d'optimisation de nc lignes et nd colonnes. Dans cette matrice, chaque ligne Ic représente une cote condition et chaque colonne Id représente une dispersion comme le montre le tableau 2 [15]. On remarque que chaque ligne correspond à une chaîne de tolérances associée à une chaîne de cotes fonctionnelles. On remplit dans chaque colonne la valeur de la dispersion repérée par la variable $x$ si celle-ci fait partie de la chaîne de tolérance. Dans le cas contraire on remplit la valeur 0 .

\subsection{Organigramme d'optimisation}

Comme le montre l'organigramme de la figure 3 , en premier lieu, on calcule le coefficient de répartition $k^{\prime} j$ pour toutes les cotes condition $C C$ par la relation (3) où $w$ représente le nombre des dispersions connues, $p$ le nombre des dispersions inconnues et $j$ le numéro d'itération.

$$
k_{j}^{\prime}=\frac{I T c c-\sum_{i=1}^{w} \Delta l i}{p}
$$


Tableau 1. Représentation matricielle de l'assemblage.

\begin{tabular}{|c|c|c|c|c|c|c|c|c|c|c|c|c|c|c|c|c|}
\hline & \multicolumn{15}{|c|}{ Surfaces } \\
\hline & & 1 & 2 & 3 & 4 & 5 & 6 & 7 & 8 & 9 & 10 & 11 & 12 & 13 & 14 & 15 \\
\hline \multirow{7}{*}{ Pièces } & $\mathrm{A}$ & 0 & $\Delta l_{2}^{(\mathrm{A})}$ & 0 & 0 & $\Delta l_{5}^{(\mathrm{A})}$ & 0 & $\Delta l_{7}^{(\mathrm{A})}$ & $\Delta l_{8}^{(\mathrm{A})}$ & $\Delta l_{9}^{(\mathrm{A})}$ & 0 & $\Delta l_{11}^{(\mathrm{A})}$ & $\Delta l_{12}^{(\mathrm{A})}$ & 0 & 0 & $\Delta l_{15}^{(\mathrm{A})}$ \\
\hline & B & $\Delta l_{1}^{(\mathrm{B})}$ & 0 & 0 & 0 & 0 & $\Delta l_{6}^{(\mathrm{B})}$ & 0 & $\Delta l_{8}^{(\mathrm{B})}$ & 0 & 0 & 0 & 0 & 0 & 0 & 0 \\
\hline & $\mathrm{C}$ & 0 & 0 & 0 & $\Delta l_{4}^{(\mathrm{C})}$ & 0 & $\Delta l_{6}^{(\mathrm{C})}$ & 0 & 0 & 0 & 0 & 0 & 0 & 0 & 0 & 0 \\
\hline & $\mathrm{D}$ & 0 & 0 & $\Delta l_{3}^{(\mathrm{D})}$ & $\Delta l_{4}^{(\mathrm{D})}$ & 0 & 0 & 0 & 0 & 0 & 0 & 0 & 0 & 0 & 0 & 0 \\
\hline & $\mathrm{E}$ & 0 & 0 & 0 & 0 & 0 & 0 & 0 & 0 & 0 & $\Delta l_{10}^{(\mathrm{E})}$ & $\Delta l_{11}^{(\mathrm{E})}$ & 0 & 0 & 0 & 0 \\
\hline & $\mathrm{F}$ & 0 & 0 & 0 & 0 & 0 & 0 & 0 & 0 & 0 & 0 & $\Delta l_{11}^{(\mathrm{F})}$ & 0 & $\Delta l_{13}^{(\mathrm{F})}$ & 0 & 0 \\
\hline & G & 0 & 0 & 0 & 0 & 0 & 0 & 0 & 0 & 0 & 0 & 0 & 0 & $\Delta l_{13}^{(\mathrm{G})}$ & $\Delta l_{14}^{(\mathrm{G})}$ & 0 \\
\hline
\end{tabular}

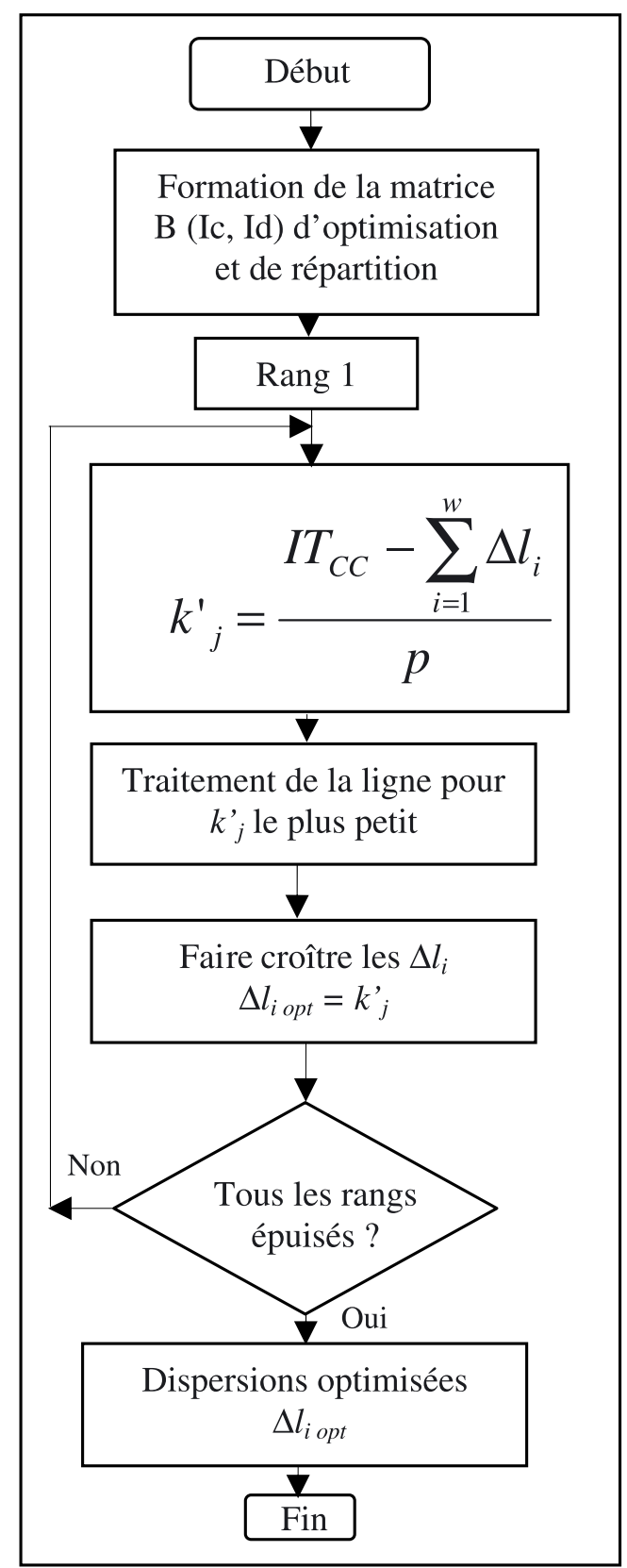

Fig. 3. Organigramme de l'optimisation des dispersions par la méthode des dispersions inconnues [14].
On commence par la ligne dont le coefficient $k^{\prime} j$ est le plus petit. Une fois que les valeurs des dispersions de la ligne sont calculées par la relation (3), nous les affichons sur toute la colonne où elles sont présentes. On recommence à calculer les nouveaux coefficients $k^{\prime} j$ avec les nouvelles $\Delta l i$ jusqu'à l'épuisement des lignes des cotes condition $C C$.

\subsection{Procédure d'optimisation}

Détermination de la ligne de traitement pour le rang 1 (Tab. 3).

D'après le tableau 3 on remarque que la ligne 5 a le plus petit coefficient $\left(k^{\prime} j=0,05\right)$, donc on va commencer par traiter cette ligne.

En traitant ligne par ligne, on va retrouver le tableau des dispersions optimisées (Tab. 4).

\section{Synthèse des cotes fonctionnelles}

La méthode des dispersions peut être utilisée pour simuler et déterminer les valeurs des cotes fonctionnelles qui répondent aux exigences fonctionnelles. Étant donné que les cotes fonctionnelles doivent être à la fin fabriquées, le modèle fondamental des cotes moyennes permet d'établir des relations entre les cotes fonctionnelles et les cotes de simulation $L i$ comme le montre la relation (4)

$$
\text { Cf } i, j=L j-L i \quad \text { avec } \quad j>i
$$

Pour positionner $n$ surfaces il faut déterminer $(n-1)$ longueurs moyennes de base [6]. Nous aurons besoin donc de $(n-1)$ équations à résoudre pour déterminer les $(n-1)$ longueurs sachant que $L_{1}=0$ (en prenant l'origine sur la première surface). Un système d'équations est ainsi construit en utilisant les valeurs moyennes des cotes condition $C C$ et cotes standard $C S$ par les relations (5) et (6). Les cotes $C S$ sont choisies pour compléter le système parmi les cotes fonctionnelles de pièces standard, par exemple de sous-traitance.

$$
\begin{aligned}
& \left(C C i_{, j}\right)_{\text {moy }}=L j-L i \\
& \left(C S i_{, j}\right)_{\text {moy }}=L j-L I
\end{aligned}
$$




\begin{tabular}{|c|c|c|c|c|c|c|c|}
\hline \pm & \begin{tabular}{|l|}
20 \\
8 \\
\end{tabular} & $\mid \begin{array}{c}10 \\
-1\end{array}$ & $\rightarrow$ & $\rightarrow$ & $\tilde{o}$ & $\rightarrow$ & 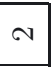 \\
\hline & 0 & 0 & 0 & 0 & 0 & 0 & $\Leftrightarrow$ \\
\hline & 0 & 0 & 0 & 0 & 0 & 0 & 8 \\
\hline & 0 & 0 & 0 & 0 & 0 & 0 & 8 \\
\hline & 0 & 0 & 0 & 0 & 0 & $\&$ & $\&$ \\
\hline & 0 & 0 & 0 & 0 & 0 & $\Leftrightarrow$ & 0 \\
\hline & 0 & 0 & 0 & 0 & 0 & $\&$ & $\Leftrightarrow$ \\
\hline & 0 & 0 & 0 & 0 & $\&$ & 0 & 0 \\
\hline & 0 & 0 & 0 & 0 & $\&$ & $\Leftrightarrow$ & 8 \\
\hline & 0 & 0 & 0 & 0 & $\&$ & 0 & 0 \\
\hline & 0 & 0 & 0 & 0 & $\&$ & 0 & 0 \\
\hline & 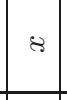 & $\&$ & $\leftrightarrow$ & $\Leftrightarrow$ & 0 & 0 & 0 \\
\hline & 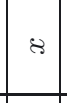 & $\&$ & $\&$ & $\Leftrightarrow$ & 0 & 0 & 0 \\
\hline & 0 & 0 & 0 & $\&$ & 0 & 0 & 0 \\
\hline & 0 & $\&$ & $\&$ & 0 & 0 & 0 & 0 \\
\hline $\begin{array}{l}0_{0} \\
\overrightarrow{7}\end{array}$ & 0 & $\&$ & $\because 1$ & $\Leftrightarrow$ & 0 & 0 & 0 \\
\hline 1 & 0 & 0 & $\&$ & 0 & 0 & 0 & 0 \\
\hline 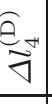 & 0 & $\&$ & 0 & 0 & 0 & 0 & 0 \\
\hline t. & 0 & $\&$ & $\&$ & 0 & 0 & 0 & 0 \\
\hline$\hat{e}_{\infty}$ & 0 & $\&$ & 0 & 0 & 0 & 0 & 0 \\
\hline$\underset{\nabla}{\mathbb{E}}$ & . & $\&$ & 0 & 0 & 0 & 0 & 0 \\
\hline 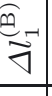 & $\approx$ & 0 & 0 & 0 & 0 & 0 & 0 \\
\hline 0 & $\stackrel{\sim}{\vec{D}}$ & $\mid \begin{array}{c}\infty \\
\stackrel{i}{i} \\
\end{array}$ & $\begin{array}{c}10 \\
0 \\
0 \\
f\end{array}$ & $\begin{array}{l}1 \\
\hat{2}\end{array}$ & \begin{tabular}{|l|l}
0 \\
3 \\
8 \\
8
\end{tabular} & \begin{tabular}{|r|}
0 \\
0 \\
1 \\
3 \\
\end{tabular} & . \\
\hline 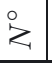 & -1 & $\sim$ & $\infty$ & + & $\infty$ & 0 & $N$ \\
\hline
\end{tabular}

\begin{tabular}{|c|c|c|c|c|c|c|c|}
\hline & $\left|\begin{array}{l}\stackrel{2}{2} \\
\stackrel{7}{0}\end{array}\right|$ & $\mid \begin{array}{l}10 \\
\infty \\
0 \\
0 \\
0\end{array}$ & $\left|\begin{array}{l}0 \\
0 \\
0 \\
0\end{array}\right|$ & $\begin{array}{l}\stackrel{2}{2} \\
0 \\
0\end{array}$ & $\begin{array}{l}12 \\
0 \\
0\end{array}$ & $\begin{array}{l}\stackrel{1}{1} \\
\text { on }\end{array}$ & 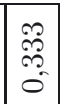 \\
\hline$E$ & \begin{tabular}{|l|}
0 \\
0
\end{tabular} & $\stackrel{10}{-10}$ & $\neg$ & $\neg$ & \begin{tabular}{|l|}
$\tilde{y}$ \\
$\tilde{0}$
\end{tabular} & - & N \\
\hline & 0 & 0 & 0 & 0 & 0 & 0 & $\Leftrightarrow$ \\
\hline & 0 & 0 & 0 & 0 & 0 & 0 & 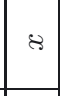 \\
\hline & 0 & 0 & 0 & 0 & 0 & 0 & $\therefore$ \\
\hline & 0 & 0 & 0 & 0 & 0 & $\because$ & $\&$ \\
\hline & 0 & 0 & 0 & 0 & 0 & $\therefore$ & 0 \\
\hline & 0 & 0 & 0 & 0 & 0 & 8 & $\&$ \\
\hline & 0 & 0 & 0 & 0 & $\&$ & 0 & 0 \\
\hline & 0 & 0 & 0 & 0 & 8 & 8 & $\&$ \\
\hline & 0 & 0 & 0 & 0 & $\&$ & 0 & 0 \\
\hline & 0 & 0 & 0 & 0 & 8 & 0 & 0 \\
\hline & $\&$ & $\Leftrightarrow$ & $\Leftrightarrow$ & $\&$ & 0 & 0 & 0 \\
\hline & $\&$ & 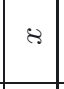 & $\Leftrightarrow$ & $\&$ & 0 & 0 & 0 \\
\hline & 0 & 0 & 0 & $\&$ & 0 & 0 & 0 \\
\hline & 0 & $\&$ & 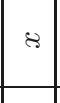 & 0 & 0 & 0 & 0 \\
\hline & 0 & $\&$ & $\&$ & $\&$ & 0 & 0 & 0 \\
\hline & 0 & 0 & $\Leftrightarrow$ & 0 & 0 & 0 & 0 \\
\hline & 0 & $\&$ & 0 & 0 & 0 & 0 & 0 \\
\hline & 0 & $\&$ & $\&$ & 0 & 0 & 0 & 0 \\
\hline & 0 & 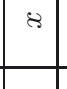 & 0 & 0 & 0 & 0 & 0 \\
\hline 7 & $\&$ & $\&$ & 0 & 0 & 0 & 0 & 0 \\
\hline$\frac{e}{z}$ & $\&$ & 0 & 0 & 0 & 0 & 0 & 0 \\
\hline 0 & $\stackrel{\sim}{\vec{J}}$ & {$\left[\begin{array}{c}\infty \\
\stackrel{1}{i}\end{array}\right.$} & \begin{tabular}{|l|}
10 \\
f \\
\end{tabular} & $\begin{array}{l}1 \\
0 \\
0\end{array}$ & \begin{tabular}{|l|}
0 \\
0 \\
8 \\
\end{tabular} & \begin{tabular}{|r|}
0 \\
0 \\
in \\
s. \\
\end{tabular} & 紫 \\
\hline 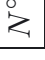 & $\mid-1$ & o & $\infty$ & 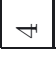 & 10 & 0 & N \\
\hline
\end{tabular}

\begin{tabular}{|c|c|c|c|c|c|c|c|}
\hline & $\mid \begin{array}{l}120 \\
0 \\
0\end{array}$ & $\mid \begin{array}{c}10 \\
\rightarrow-1\end{array}$ & $\rightarrow$ & $\rightarrow$ & $\tilde{\sigma}$ & $\rightarrow$ & $\curvearrowright$ \\
\hline & 0 & 0 & 0 & 0 & 0 & 0 & $\begin{array}{l}\stackrel{9}{9} \\
\stackrel{9}{9} \\
0\end{array}$ \\
\hline & 0 & 0 & 0 & 0 & 0 & 0 & 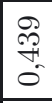 \\
\hline & 0 & 0 & 0 & 0 & 0 & 0 & 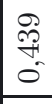 \\
\hline & 0 & 0 & 0 & 0 & 0 & $\begin{array}{l}0 \\
m \\
\vdots \\
0\end{array}$ & $\begin{array}{l}0 \\
0 \\
0 \\
\end{array}$ \\
\hline & 0 & 0 & 0 & 0 & 0 & $\begin{array}{l}0 \\
0 \\
0 \\
0\end{array}$ & 0 \\
\hline & 0 & 0 & 0 & 0 & 0 & $\begin{array}{c}0 \\
m \\
0 \\
0\end{array}$ & $\begin{array}{l}0 \\
m \\
0 \\
0\end{array}$ \\
\hline & 0 & 0 & 0 & 0 & $\begin{array}{l}28 \\
0 \\
0\end{array}$ & 0 & 0 \\
\hline & 0 & 0 & 0 & 0 & $\begin{array}{l}20 \\
0 \\
0\end{array}$ & $\begin{array}{l}28 \\
0 \\
0\end{array}$ & $\stackrel{28}{0}$ \\
\hline & 0 & 0 & 0 & 0 & $\begin{array}{l}28 \\
0 \\
0\end{array}$ & 0 & 0 \\
\hline & 0 & 0 & 0 & 0 & $\begin{array}{l}20 \\
0 \\
0\end{array}$ & 0 & 0 \\
\hline & $\mid \begin{array}{c}2 \\
\stackrel{2}{0} \\
0\end{array}$ & $\begin{array}{c}\stackrel{2}{1} \\
\stackrel{0}{0}\end{array}$ & $\begin{array}{c}20 \\
\stackrel{2}{0} \\
0\end{array}$ & $\begin{array}{l}20 \\
\\
0\end{array}$ & 0 & 0 & 0 \\
\hline & $\begin{array}{c}2 \\
\stackrel{2}{7} \\
0\end{array}$ & $\begin{array}{c}\stackrel{2}{0} \\
\stackrel{7}{0} \\
0\end{array}$ & $\begin{array}{c}2 \\
\stackrel{2}{7} \\
0 \\
0\end{array}$ & $\begin{array}{c}2 \\
0 \\
0 \\
0\end{array}$ & 0 & 0 & 0 \\
\hline & 0 & 0 & 0 & \begin{tabular}{|c|c} 
& 0 \\
6 \\
0 \\
0 \\
\end{tabular} & 0 & 0 & 0 \\
\hline & 0 & $\begin{array}{l}1 \\
\infty \\
0 \\
0\end{array}$ & $\mid \begin{array}{c}1 \\
\infty \\
0 \\
0\end{array}$ & 0 & 0 & 0 & 0 \\
\hline & 0 & $\begin{array}{l}1 \\
\infty \\
0 \\
0 \\
\end{array}$ & \begin{tabular}{|c|c}
1 \\
$\infty$ \\
-1 \\
0 \\
\end{tabular} & $\begin{array}{l}1 \\
\infty \\
0 \\
0 \\
\end{array}$ & 0 & 0 & 0 \\
\hline & 0 & 0 & \begin{tabular}{|c|c|}
1 \\
0 \\
0 \\
0 \\
\end{tabular} & 0 & 0 & 0 & 0 \\
\hline & 0 & 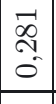 & 0 & 0 & 0 & 0 & 0 \\
\hline & 0 & $\begin{array}{l}1 \\
\infty \\
0 \\
0\end{array}$ & $\mid \begin{array}{c}1 \\
\infty \\
0 \\
0 \\
0\end{array}$ & 0 & 0 & 0 & 0 \\
\hline & & 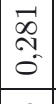 & 0 & 0 & 0 & 0 & 0 \\
\hline & $\begin{array}{c}2 \\
\stackrel{1}{1} \\
0 \\
\end{array}$ & $\begin{array}{l}2 \\
\stackrel{1}{0} \\
0 \\
\end{array}$ & 0 & 0 & 0 & 0 & 10 \\
\hline & $\mid \begin{array}{c}2 \\
\stackrel{9}{1} \\
0\end{array}$ & 0 & 0 & 0 & 0 & 0 & 0 \\
\hline$=$ & $\begin{array}{l}7 \\
\vec{D}\end{array}$ & 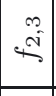 & \begin{tabular}{|l|}
0 \\
fi \\
\end{tabular} & $\begin{array}{l}1 \\
0 \\
0\end{array}$ & $\begin{array}{l}0 \\
3 \\
8 \\
8\end{array}$ & 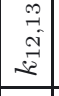 & 资 \\
\hline & -1 & $\sim$ & $\infty$ & 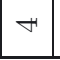 & 10 & 0 & \\
\hline
\end{tabular}


où $i$ et $j$ sont les surfaces qui délimitent les cotes condition $C C$ ou $C S$.

Dans le cas de cotes unilimites, $C C_{\text {moy }}$ et $C S_{\text {moy }}$ sont calculées en utilisant l'intervalle de tolérance optimisé par les relations suivantes :

$$
\begin{aligned}
C C_{\mathrm{moy}} & =\frac{C C_{\min }+C C_{\max }}{2} \\
C S_{\mathrm{moy}} & =\frac{C S_{\min }+C S_{\max }}{2}
\end{aligned}
$$

Les équations (5) à (8) donnent un système de $n$ équations à $n$ inconnues $L i$. Une fois que les longueurs de simulation $L i$ sont trouvées, on calcule les cotes fonctionnelles moyennes par la relation (4).

- La matrice élargie après optimisation des dispersions à l'aide des IT des cotes conditions bilatérales pour cet exemple est donnée comme suit (Tab. 5).

- Calcul des longueurs moyennes de base Li.

Pour cet exemple nous avons 15 surfaces alors nous aurons besoin de 14 cotes conditions. On complète donc les 7 exigences fonctionnelles $e, f, g, h, q, k$ et $j$ par 7 autres cotes conditions comme cotes mini. Celles-ci sont données par les pièces standards ou de sous-traitance.

Pour notre cas, on prend :

l'écrou $\mathrm{D}\left(C S_{3,4}^{(\mathrm{D})}=10 \mathrm{mini}\right)$;

la rondelle $\mathrm{C}\left(C S_{4,6}^{(\mathrm{C})}=4 \mathrm{mini}\right)$;

la pièce $\mathrm{B}\left(C S_{1,8}^{(\mathrm{B})}=40 \mathrm{mini}\right)$;

le côté gauche de l'arbre $\mathrm{A}\left(C S_{8,9}^{(\mathrm{A})}=5 \mathrm{mini}\right)$;

la pièce $\mathrm{E}\left(C S_{10,11}^{(\mathrm{E})}=16 \mathrm{mini}\right)$;

la rondelle $\mathrm{C}\left(C S_{11,13}^{(\mathrm{F})}=4 \mathrm{mini}\right)$ et

l'écrou D $\left(C S_{13,14}^{(\mathrm{G})}=10 \mathrm{mini}\right)$.

Pour les 15 surfaces, il y aura 15 longueurs moyennes de base inconnues à déterminer (donc 15 équations à résoudre, avec $L_{1}=0$ ).

Les équations des conditions bilimites sont données par :

Équation $1: e_{1,2}=L_{2}-L_{1}=2$

Équation $2: f_{2,3}=L_{3}-L_{2}=4$

Équation $3: g_{4,5}=L_{5}-L_{4}=2$

Équation $4: h_{6,7}=L_{7}-L_{6}=2$

Équation $7: q_{9,10}=L_{10}-L_{9}=3$

Équation $5: k_{12,13}=L_{13}-L_{12}=2$

Équation $6: j_{14,15}=L_{15}-L_{14}=4$.

Les équations des conditions unilimites sont données par :

Équation $8: C S_{3,4}=L_{4}-L_{3}=10,281$

Équation 9 : $C S_{4,6}=L_{6}-L_{4}=4,187$

Équation $10: C S_{1,8}=L_{8}-L_{1}=40,125$

Équation $11: C S_{8,9}=L_{9}-L_{8}=5,088$

Équation $12: C S_{10,11}=L_{11}-L_{10}=16,05$

\begin{tabular}{|c|c|c|c|c|c|c|c|c|c|c|c|c|c|c|}
\hline $\begin{array}{l}\ddot{\tilde{\nabla}} \\
\tilde{\sigma}\end{array}$ & 10 & $\stackrel{L}{-10}$ & $\neg$ & $\neg$ & $\tilde{o}$ & $\neg$ & N & 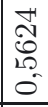 & $\begin{array}{c}10 \\
1 \\
0 \\
0 \\
0\end{array}$ & 尽 & $\begin{array}{l}10 \\
\stackrel{1}{二} \\
2 \\
0\end{array}$ & $\Rightarrow$ & 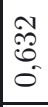 & $\begin{array}{l}\infty \\
1 \\
\infty \\
0 \\
0\end{array}$ \\
\hline$\Xi$ & $\begin{array}{l}10 \\
0\end{array}$ & 10 & $\dashv$ & $\neg$ & $\tilde{O}$ & -1 & N & 1 & 1 & 1 & 1 & 1 & 1 & 1 \\
\hline$\stackrel{4}{\overrightarrow{4}}$ & 0 & 0 & 0 & 0 & 0 & 0 & 审 & 0 & 0 & 0 & 0 & 0 & 0 & 0 \\
\hline$\stackrel{\overrightarrow{7}}{\overrightarrow{7}}$ & 0 & 0 & 0 & 0 & 0 & 0 & 今. & 0 & 0 & 0 & 0 & 0 & 0 & 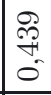 \\
\hline 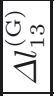 & 0 & 0 & 0 & 0 & 0 & 0 & 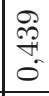 & 0 & 0 & 0 & 0 & 0 & 0 & 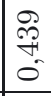 \\
\hline$\underset{\nabla}{\vec{\nabla}}$ & 0 & 0 & 0 & 0 & 0 & $\begin{array}{l}0 \\
0 \\
0 \\
0\end{array}$ & $\begin{array}{l}0 \\
\stackrel{0}{n} \\
0 \\
0\end{array}$ & 0 & 0 & 0 & 0 & 0 & 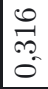 & 0 \\
\hline$\frac{\vec{y}}{\overrightarrow{7}}$ & 0 & 0 & 0 & 0 & 0 & $\begin{array}{l}0 \\
-3 \\
0 \\
0\end{array}$ & 0 & 0 & 0 & 0 & 0 & 0 & 0 & 0 \\
\hline$\vec{\nabla}$ & 0 & 0 & 0 & 0 & 0 & $\begin{array}{l}0 \\
-1 \\
0 \\
0\end{array}$ & $\begin{array}{l}0 \\
0 \\
0 \\
0 \\
0\end{array}$ & 0 & 0 & 0 & 0 & 0 & $\begin{array}{l}0 \\
\mathscr{\sigma} \\
\tilde{\sigma} \\
0\end{array}$ & 0 \\
\hline$\stackrel{\sqrt{\mathbf{I}}}{\overrightarrow{7}}=$ & 0 & 0 & 0 & 0 & $\begin{array}{l}2 \\
0 \\
0 \\
0\end{array}$ & 0 & 0 & 0 & 0 & 0 & 0 & 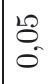 & 0 & 0 \\
\hline $\begin{array}{l}\stackrel{4}{\vec{Z}} \\
\vec{\nabla}\end{array}$ & 0 & 0 & 0 & 0 & $\begin{array}{l}2 \\
0 \\
0\end{array}$ & $\begin{array}{l}12 \\
0 \\
0\end{array}$ & $\stackrel{20}{0}$ & 0 & 0 & 0 & 0 & 0 & 0 & 0 \\
\hline 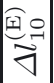 & 0 & 0 & 0 & 0 & $\begin{array}{l}12 \\
0 \\
0\end{array}$ & 0 & 0 & 0 & 0 & 0 & 0 & $\stackrel{12}{2}$ & 0 & 0 \\
\hline$\underset{\forall}{\mathbb{J}} \sigma$ & 0 & 0 & 0 & 0 & 20. & 0 & 0 & 0 & 0 & 0 & $\left|\begin{array}{l}12 \\
0 \\
0\end{array}\right|$ & 0 & 0 & 0 \\
\hline $\begin{array}{l}\hat{\theta}_{\infty} \\
\vec{\nabla}\end{array}$ & 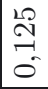 & $\begin{array}{l}\stackrel{L}{\sim} \\
\stackrel{-}{I} \\
0\end{array}$ & 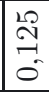 & 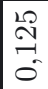 & 0 & 0 & 0 & 0 & 0 & 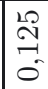 & 0 & 0 & 0 & 0 \\
\hline$\underset{\forall}{\infty}$ & 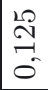 & $\begin{array}{l}\stackrel{1}{\mathcal{I}} \\
\stackrel{-}{0}\end{array}$ & 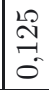 & 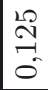 & 0 & 0 & 0 & 0 & 0 & 0 & $\begin{array}{l}1 \\
\sim \\
\mathcal{1} \\
0 \\
0\end{array}$ & 0 & 0 & 0 \\
\hline $\begin{array}{l}\vec{E}_{n} \\
\vec{\nabla}\end{array}$ & 0 & 0 & 0 & 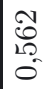 & 0 & 0 & 0 & 0 & 0 & 0 & 0 & 0 & 0 & 0 \\
\hline $\begin{array}{l}\vec{v}_{0} \\
\vec{\nabla}\end{array}$ & 0 & 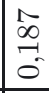 & $\begin{array}{c}1 \\
\infty \\
-1 \\
0 \\
0\end{array}$ & 0 & 0 & 0 & 0 & 0 & $\mid \begin{array}{l}1 \\
\infty \\
0 \\
0\end{array}$ & 0 & 0 & 0 & 0 & 0 \\
\hline $\begin{array}{l}\tilde{e}_{0} \\
\vec{\nabla}\end{array}$ & 0 & 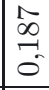 & $\mid \begin{array}{c}1 \\
\infty \\
-1 \\
0 \\
0\end{array}$ & $\mid \begin{array}{l}1 \\
\infty \\
-1 \\
0 \\
0\end{array}$ & 0 & 0 & 0 & 0 & 0 & 0 & 0 & 0 & 0 & 0 \\
\hline $\mid \begin{array}{l}\vec{Z}_{10} \\
\vec{\nabla}\end{array}$ & 0 & 0 & 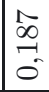 & 0 & 0 & 0 & 0 & 0 & 0 & 0 & 0 & 0 & 0 & 0 \\
\hline $\overrightarrow{\hat{\theta}}_{\overrightarrow{+}}$ & 0 & \begin{tabular}{l}
$\vec{\infty}$ \\
\multirow{N}{N}{} \\
0
\end{tabular} & 0 & 0 & 0 & 0 & 0 & $\begin{array}{l}\vec{\infty} \\
\text { N } \\
\tilde{0}\end{array}$ & 0 & 0 & 0 & 0 & 0 & 0 \\
\hline 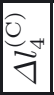 & 0 & $\begin{array}{l}1 \\
\infty \\
-1 \\
0\end{array}$ & $\begin{array}{l}1 \\
\infty \\
-1 \\
0\end{array}$ & 0 & 0 & 0 & 0 & 0 & 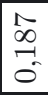 & 0 & 0 & 0 & 0 & 0 \\
\hline$\hat{\theta}_{\infty}$ & 0 & $\begin{array}{l}\vec{\infty} \\
\stackrel{N}{N} \\
0\end{array}$ & 0 & 0 & 0 & 0 & 0 & $\begin{array}{l}\vec{\infty} \\
\stackrel{\infty}{N} \\
0\end{array}$ & 0 & 0 & 0 & 0 & 0 & 0 \\
\hline $\mid$ & $\begin{array}{l}10 \\
\stackrel{1}{2} \\
\stackrel{-}{0} \\
0\end{array}$ & 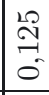 & 0 & 0 & 0 & 0 & 0 & 0 & 0 & 0 & 0 & 0 & 0 & 0 \\
\hline$\underset{\vec{\nabla}}{\vec{\theta}}$ & $\begin{array}{l}10 \\
\stackrel{10}{7} \\
0 \\
0\end{array}$ & 0 & 0 & 0 & 0 & 0 & 0 & 0 & 0 & $\begin{array}{l}10 \\
\stackrel{10}{7} \\
0 \\
0\end{array}$ & 0 & 0 & 0 & 0 \\
\hline 0 & $\stackrel{\overbrace{}}{\mathcal{J}}$ & 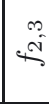 & $\stackrel{2}{8}$ & ¿̊ & $\begin{array}{l}\circ \\
\text { के }\end{array}$ & $\begin{array}{l}m \\
\overrightarrow{7} \\
\overrightarrow{3} \\
3\end{array}$ & $\stackrel{20}{\stackrel{2}{+}}$ & $\begin{array}{l}\hat{e}_{\tilde{c}}^{+} \\
\sigma^{\infty}\end{array}$ & 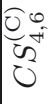 & $\mid \begin{array}{l}\infty \\
\tilde{c}^{\infty} \\
u\end{array}$ & $\mid \begin{array}{c}\int_{0} \\
\omega_{0} \\
\tau^{2}\end{array}$ & $\begin{array}{l}17 \\
\underbrace{7} 0 \\
0 \\
0 \\
0\end{array}$ & 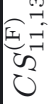 & $\begin{array}{l}0 \\
0 \\
v^{2} \\
\sigma^{2}\end{array}$ \\
\hline 吕 & $\neg$ & $\sim$ & $\infty$ & 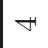 & ס & 0 & $N$ & $\infty$ & 0 & $\circ$ & $\exists$ & $\stackrel{\mathcal{Y}}{\sim}$ & $\dddot{m}$ & $\exists$ \\
\hline
\end{tabular}

Équation 13 : $C S_{11,13}=L_{13}-L_{11}=4,317$ 
Tableau 6. Système d'équations linéaires.

$$
\left[\begin{array}{ccccccccccccccc}
-1 & 1 & 0 & 0 & 0 & 0 & 0 & 0 & 0 & 0 & 0 & 0 & 0 & 0 & 0 \\
0 & -1 & 1 & 0 & 0 & 0 & 0 & 0 & 0 & 0 & 0 & 0 & 0 & 0 & 0 \\
0 & 0 & 0 & -1 & 1 & 0 & 0 & 0 & 0 & 0 & 0 & 0 & 0 & 0 & 0 \\
0 & 0 & 0 & 0 & 0 & -1 & 1 & 0 & 0 & 0 & 0 & 0 & 0 & 0 & 0 \\
0 & 0 & 0 & 0 & 0 & 0 & 0 & 0 & -1 & 1 & 0 & 0 & 0 & 0 & 0 \\
0 & 0 & 0 & 0 & 0 & 0 & 0 & 0 & 0 & 0 & 0 & -1 & 1 & 0 & 0 \\
0 & 0 & 0 & 0 & 0 & 0 & 0 & 0 & 0 & 0 & 0 & 0 & 0 & -1 & 1 \\
0 & 0 & -1 & 1 & 0 & 0 & 0 & 0 & 0 & 0 & 0 & 0 & 0 & 0 & 0 \\
0 & 0 & 0 & -1 & 0 & 1 & 0 & 0 & 0 & 0 & 0 & 0 & 0 & 0 & 0 \\
-1 & 0 & 0 & 0 & 0 & 0 & 0 & 1 & 0 & 0 & 0 & 0 & 0 & 0 & 0 \\
0 & 0 & 0 & 0 & 0 & 0 & 0 & -1 & 1 & 0 & 0 & 0 & 0 & 0 & 0 \\
0 & 0 & 0 & 0 & 0 & 0 & 0 & 0 & 0 & -1 & 1 & 0 & 0 & 0 & 0 \\
0 & 0 & 0 & 0 & 0 & 0 & 0 & 0 & 0 & 0 & -1 & 0 & 1 & 0 & 0 \\
0 & 0 & 0 & 0 & 0 & 0 & 0 & 0 & 0 & 0 & 0 & 0 & -1 & 1 & 0 \\
1 & 0 & 0 & 0 & 0 & 0 & 0 & 0 & 0 & 0 & 0 & 0 & 0 & 0 & 0
\end{array}\right]\left[\begin{array}{c}
L_{1} \\
L_{2} \\
L_{3} \\
L_{4} \\
L_{5} \\
L_{6} \\
L_{7} \\
L_{8} \\
L_{9} \\
L_{10} \\
L_{11} \\
L_{12} \\
L_{13} \\
L_{14} \\
L_{15}
\end{array}\right]=\left[\begin{array}{c}
2 \\
4 \\
2 \\
2 \\
3 \\
2 \\
4 \\
10,281 \\
4,187 \\
40,125 \\
5,088 \\
16,05 \\
4,317 \\
10,439 \\
0
\end{array}\right]
$$

Équation $14: C S_{13,14}=L_{14}-L_{13}=10,439$

Et comme équation $15: L_{15}=0$.

À partir de ces équations, on forme le système d'équations linéaires (Tab. 6).

Après résolution du système nous obtenons les valeurs suivantes pour les longueurs moyennes :

$$
\begin{array}{lcc}
L_{1}=0 & L_{2}=2 & L_{3}=6 \\
L_{4}=16,281 & L_{5}=18,281 & L_{6}=20,468 \\
L_{7}=22,468 & L_{8}=40,125 & L_{9}=45,213 \\
L_{10}=48,213 & L_{11}=64,263 & L_{12}=66,535 \\
L_{13}=68,535 & L_{14}=78,974 & L_{15}=82,974
\end{array}
$$

Ceci nous permet par la suite de calculer les cotes fonctionnelles moyennes

$$
\begin{aligned}
& C f_{1,8 \text { moy }}=L_{8}-L_{1}=40,125 \\
& C f_{2,8 \text { moy }}=L_{8}-L_{2}=38,125 \\
& C f_{6,8 \text { moy }}=L_{8}-L_{6}=19,657 \\
& C f_{3,4 \text { moy }}=L_{4}-L_{3}=10,281 \\
& C f_{4,6 \text { moy }}=L_{6}-L_{4}=4,187 \\
& C f_{5,8 \text { moy }}=L_{8}-L_{5}=21,844 \\
& C f_{7,8 \text { moy }}=L_{8}-L_{7}=17,657 \\
& C f_{8,9 \text { moy }}=L_{9}-L_{8}=5,088 \\
& C f_{9,11 \text { moy }}=L_{11}-L_{9}=19,05 \\
& C f_{10,11 \text { moy }}=L_{10}-L_{11}=16,05 \\
& C f_{11,15 \text { moy }}=L_{15}-L_{11}=18,761 \\
& C f_{11,12 \text { moy }}=L_{12}-L_{11}=2,317 \\
& C f_{11,13 \text { moy }}=L_{13}-L_{11}=4,317 \\
& C f_{13,14 \text { moy }}=L_{14}-L_{13}=10,439
\end{aligned}
$$

Calcul des cotes fonctionnelles avec leurs intervalles de tolérances

$$
\begin{aligned}
& C f_{1,8}=40,125 \pm 0,125 \\
& C f_{2,8}=38,125 \pm 0,125 \\
& C f_{6,8}=19,657 \pm 0,156 \\
& C f_{3,4}=10,281 \pm 0,281 \\
& C f_{4,6}=4,187 \pm 0,187 \\
& C f_{5,8}=21,844 \pm 0,156
\end{aligned}
$$

$$
\begin{aligned}
& C f_{7,8}=17,657 \pm 0,343 \\
& C f_{8,9}=5,088 \pm 0,088 \\
& C f_{9,11}=19,05 \pm 0,050 \\
& C f_{10,11}=16,05 \pm 0,050 \\
& C f_{11,15}=18,761 \pm 0,244 \\
& C f_{11,12}=2,317 \pm 0,183 \\
& C f_{11,13}=4,317 \pm 0,317 \\
& C f_{13,14}=10,439 \pm 0,439
\end{aligned}
$$

\section{Programmation et tests de validation}

Un module a été programmé sur la base des organigrammes précédemment donnés. Ce programme est nommé OTF (outil de tolérancement fonctionnel). Il a été programmé sous langage Visual Basic. Le programme a été testé et validé sur plusieurs exemples donnant des résultats concordant avec la simulation manuelle. Nous allons appliquer le programme OTF à l'assemblage (Fig. 1). L'écran de la figure 4, représente le fichier de données de l'assemblage, et l'écran de la figure 5, illustre le fichier des résultats.

\section{Conclusion et perspectives}

L'objectif de ce travail est de contribuer au développement d'un outil CFAO de synthèse des cotes fonctionnelles exprimées par les cotes moyennes et les intervalles de tolérance centrés, en utilisant la méthode des dispersions. Cette dernière a fait ses preuves dans la simulation des avant-projets de fabrication en optimisant les tolérances et en vérifiant la faisabilité des gammes d'usinage [13]. Un module nommé OTF (outil de tolérancement fonctionnel) a été réalisé. L'automatisation consiste premièrement en l'extraction automatique des chaînes (cotes et tolérances) fonctionnelles. Ensuite une procédure d'optimisation des dispersions est effectuée. 


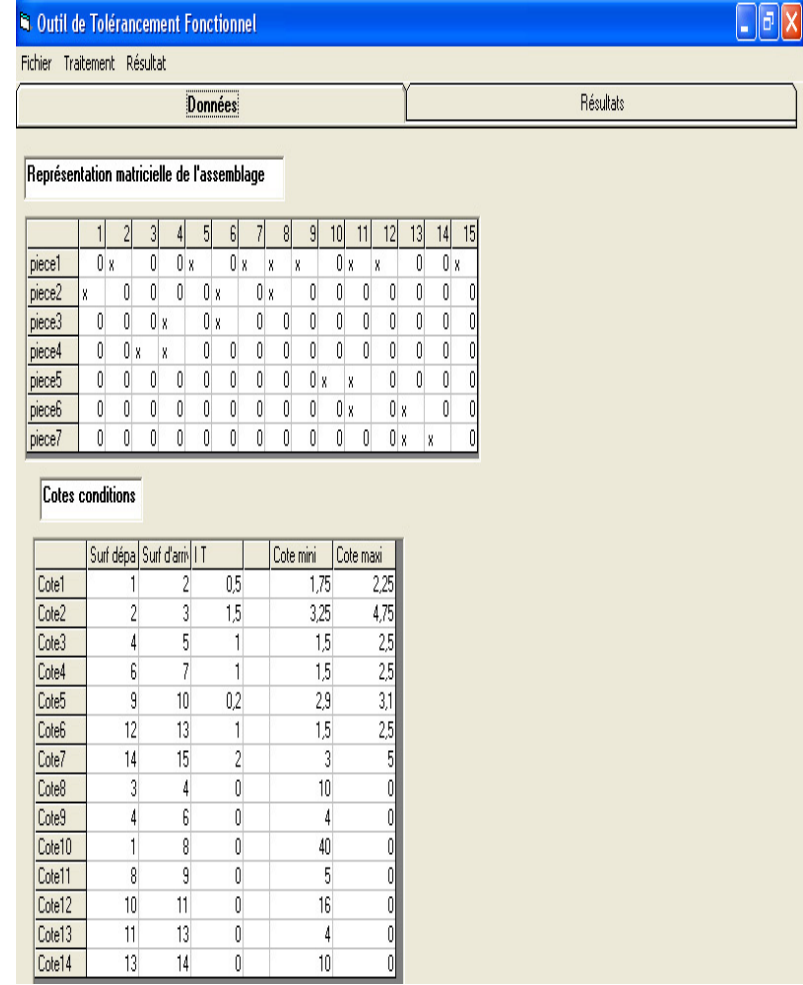

Fig. 4. Fichier de données de l'assemblage.

\begin{tabular}{|c|c|c|c|c|c|}
\hline southl & Toléraceen & Aent Fonce & actionnel & & \\
\hline Fictier TI & ennent Résalts; & & & & \\
\hline & & & smoess & & Résullats \\
\hline & sourd dépalt & sulf arivee & Colem noy & & \\
\hline Colel & 1 & & 840,125 & 0.125 & \\
\hline Colete2 & 2 & & $8 \quad 33,125$ & 0,125 & \\
\hline Coles & 3 & 4 & $\begin{array}{lll}4 & 10,281\end{array}$ & 0,281 & \\
\hline Colet & 4 & & $\begin{array}{ll}6 & 4,188\end{array}$ & 0,188 & \\
\hline Coles & 6 & & $8 \quad 196565$ & 0,156 & \\
\hline Cote6 & 5 & & $8 \quad 21,844$ & 0,156 & \\
\hline Colde? & 7 & & $8 \quad 17,556$ & 0,344 & \\
\hline Coles & 9 & 11 & $11 \quad 19,05$ & 0,05 & \\
\hline Colteg & 10 & & $11 \quad 16,055$ & 0,05 & \\
\hline Cotel10 & 11 & 12 & $\begin{array}{ll}12 & 2,317\end{array}$ & 0,183 & \\
\hline Coldel1 & 11 & & $\begin{array}{ll}13 & 4,317\end{array}$ & 0,317 & \\
\hline Colet212 & 11 & 15 & $15 \quad 18,756$ & 0.244 & \\
\hline Codet3 & 13 & 14 & $14 \quad 10,4999$ & 0,493 & \\
\hline Codel14 & 8 & 9 & $\begin{array}{lrl}9 & 5,087\end{array}$ & 0,088 & \\
\hline
\end{tabular}

Fig. 5. Fichier des résultats de l'assemblage.

Enfin les cotes fonctionnelles sont synthétisées à partir des dispersions optimisées en terme de cotes moyennes et intervalles de tolérance centrés, pour être en adéquation avec l'écriture au sens de la norme ISO des cotes fonctionnelles. En perspectives, l'intégration du module OTF dans un système CFAO permettra d'avoir automatiquement les cotes fonctionnelles exprimées par les cotes moyennes et les intervalles de tolérance centrés comme résultat final.

\section{Références}

[1] C. Marty, J.M. Linares, Industrialisation des produits mécaniques, conception et industrialisation, Publication Hermes, Tome 1, 1999

[2] L. Mathieu, A. Clement, P. Bourdet, Modelling, Representation and Processing of Tolerances, Tolerance Inspection: a survey of current hypothesis, Geometric Design Tolerancing: Theories, standards and Applications, H.A. Elmaraghy (ed.), ISBN 041283000 0, 1998, Chapman \& Hall, London, pp. 1-33

[3] J. Shah, J. Davidson, A comparative study of tolerance analysis methods, ASME Design Engineering Technical Conference and Computers and information in Engineering Conference, Salt Lake City, Utah, September 28 to October 2, 2004

[4] L. Mathieu, O. Belingard, Modélisation par les surfaces fonctionnelles en CAO : une solution pour l'intégration du tolérancement dans la maquette numérique des produits, CPI Casablanca Maroc, 2005

[5] D. Gaunet, Modèle formel de tolérancement de position. Contributions à l'aide au tolérancement des mécanismes en CFAO, Thèse de Doctorat de Mécanique, École Normale Supérieure de Cachan, 1994

[6] P. Bourdet, Chaînes de cotes de fabrication, Revue l'Ingénieur et le Technicien de l'Enseignement Technique 191 (1973) 15-23

[7] B. Anselmetti, Tolérancement : Langage des normes ISO de cotation, Édition Lavoisier, Tome 2003, 3

[8] P. Bourdet, Chaînes de cotes unidirectionnelles, ENS Cachan, 2003

[9] L. Mathieu, F. Villeneuve, Tolérancement géométrique des produits, Traité IC2, série productique, Lavoisier librairie, Hermes - Science, mai 2007

[10] D. Duret, Simulation de gamme d'usinage, Revue l'Ingénieur et le Technicien de l'Enseignement Technique 229 (1981) 34-37

[11] S. Hamou, A. Cheikh, J.M. Linares, A. Benamar, Machining dispersions based procedures for computer aided process plan simulation, Int. J. Comp. Integr. Manufact. London 17 (2004) 141-150

[12] S. Hamou, D. Moulai-Khatir, F. Hadjoui, J.M. Linares, Interfacing of unidirectional manufacturing dimensions in standardized specifications, Second International Congress Design and Modelling of Mechanical Systems (CMSM'07), 19-21 Marsh 2007, Hammamet, Tunisia

[13] S. Hamou, A. Cheikh, J.M. Linares, A. Chikh Daho, A stochastic concept for the optimization of manufacturing tolerances in computer aided process plan simulation, Int. J. Comp. Integr. Manufact. London 19 (2006) 663-675

[14] N. Medjadi-Cheikh, A. Cheikh, E. Mami, S. Hamou, Simulation des tolérances fonctionnelles : Modélisation par la méthode des dispersions, CPI Casablanca Maroc, 2005

[15] S. Hamou, Contribution à l'optimisation de la spécification des états intermédiaires dans le processus de fabrication, Thèse de Doctorat, Université de Tlemcen, Algérie, juin 2004 